\title{
Solitary Pigmented Melanocytic Lesion on the Palatal and Gingival Mucosa: Should It Rise Dentist's Concerns?
}

\author{
Cut Mytha Fitriana ${ }^{1 *}$, Yuniardini Septorini Wimardhani ${ }^{2}$ \\ 1. Oral Medicine Residency Program, Faculty of Dentistry, Universitas Indonesia, Jakarta 10430, Indonesia \\ 2. Department of Oral Medicine, Faculty of Dentistry, Universitas Indonesia, Jakarta 10430, Indonesia
}

*E-mail: mytha.fitriana@gmail.com

\begin{abstract}
Background: Solitary pigmented melanocytic lesions of the oral mucosa are sometimes presented asymptomatically. Therefore, the patient does not realize their presence. To establish a diagnosis is challenging since there are similarities with other lesions. Once pigmented lesions on the oral mucosa are found, we need to do a proper diagnosis pathway to rule out the possibility of missing the detection of a malignant lesion. This report presents a case of pigmented oral mucosa located on the palatal and gingival mucosa that had to raise the suspicion to malignant pigmented lesion.
\end{abstract}

Case Report and Management: A 46-years-old female with an asymptomatic dark bluish hue at the right hard palate towards the soft palate with a purplish at the 48-retro molar pad area for ten years ago, until a family general dentist concerned about the lesion and made the referral to our Dental Hospital. Based on the history and clinical assessment of the lesion using a diagnostic pathway, a provisional diagnosis of oral malignant melanoma was made. The biopsy for histopathological evaluation was decided. However, some obstacles prevent the patient to do the procedure. The differences in the level of awareness about pigmented lesion in the oral mucosa of the dentists involve in this patient care complicated the process of diagnosis of the lesion.

Conclusion: The dentist's awareness on the possibility of finding a nonbenign lesion in the oral cavity is needed. It is important for the dentist to make a diagnosis pathway when finding pigmented oral mucosal lesion.

Keywords: melanin, oral malignant melanoma, oral mucosa pigmentation

\section{Background}

A typical pink color with slight variation is the common color of a healthy oral of vascularization and influenced by the type of underlying connective tissue., ${ }^{1,2}$ Although some oral and perioral pigmentation may be resulted from a physiological process, some diseases or condition could manifest a various discoloration such as brown, blue, gray and black. The color changes of the oral mucosa could be resulted from various exogenous or endogenous pigmented agents. ${ }^{2}$

The exogenous pigmentation in the oral mucosa can be associated with the presence of traumatic event to the oral mucosa causing direct deposition of exogenous material to the mucosal tissues. The color changes could be resulted from deposition of metal and certain graphite, certain bacteria, medication-related or some plant derivate. The most common cause of endogenous color changes in the oral mucosa is hemoglobin, hemosiderin and melanin followed by vascular- and bilirubin-related oral pigmentation. $^{3}$ There are various manifestations of pigmentation in the oral cavity. The lesions could be a solitary to diffuse, small to large patches with a different number, distribution, shape and size, location, hue, and onset. All the manifestations would be informative for making the diagnosis. In making a proper diagnosis, besides the description of the natures of the lesion, a comprehensive history related to social, family, medical and dental issues and adjunctive examination are required. $^{2}$

Pigmented melanocytic lesion in the oral mucosa may occur physiological or pathologic. The term of melanosis is used to describe melanin over productive or hyperpigmentation. The incidence of intra-oral hyperpigmentation can occur as a physiological process; developmental; certain medication uses such oral contraceptives, high serum concentrations of ACTH (adrenocorticotropic hormone); post inflammatory changes, genetic or autoimmune disease, a neoplastic condition or idiopathic. ${ }^{3,4}$ It is important for a dentist to investigate the possible causes of the oral hyperpigmentation regarding the presence or absence systemic signs and symptoms. The decision to plan for a tissue biopsy is important for the lesions which suspected 
etiology of the hyperpigmentation could not be warranted. The decision is important to rule out the oral malignant melanoma (OMM) which could appear as a clinically benign lesion. 2

To be able to make the proper decision, a dentist should do a thorough history taking, do a clinical examination and do a series of decision making through good algorithms for pigmented lesion. Here, we described a focal pigmented lesion on the palatal mucosa of a 46-year old female patient which local and systemic signs and symptoms could not be revealed. We emphasize the importance to raise the dentist awareness of the presence of such lesion and the importance to make a decision for biopsy in making a definitive diagnosis.

\section{Case Report}

A 46-years-old female patient was referred to the Oral Medicine Clinic of the Dental Hospital, Faculty of Dentistry, Universitas Indonesia. She was referred by a general dentist who was doing a root canal treatment for the 46 tooth. The dentist noticed an asymptomatic dark bluish pigmented lesion on hard palate area of the patient.

The history taking revealed that the patient had been noticing the color changes on the palate for about ten years. A review of the medical history revealed a history of hysterectomy about eight years before the visit, and the patient had no menstrual period since. The patient did not remember any information related to her history of hysterectomy. Otherwise, the patient was clinically healthy and was not under any medication. The intraoral examination showed fair oral hygiene, with a few sub and supra-gingival calculus, nonvital 36 tooth with mobility grades 2, with anterior teeth crowding, 26 had GIC fillings, and 46 had temporary filling and loss of 35 tooth. On the palatal mucosa, there were several solitary pigmented lesions. Areas of irregular dark blue to purplish macular lesion situated between the borders of the hard palate posterior to the soft palate, measuring $10 \times 12 \mathrm{~mm}$. Secondly, several macular lesions were found on the upright the palate that was close to the midline. They were conveniently located in the part of the soft palate with a bluish to purplish color, measuring $7 \times 12 \mathrm{~mm}$. At the right distal retromolar pad area of 48 tooth, there were some dark purplish-smooth surface soft nodules, measuring about $5 \times 10 \mathrm{~mm}$. The nodules were not tender on palpation. (Figure 1).

Based on the history and clinical picture of the lesion, the provisional diagnosis of OMM with differential diagnosis of melanoacanthoma and hemangioma were suspected. The patient was asked to do a complete blood count (CBC) examination, an orthopantomogram (OPG) and an occlusal radiograph. The results of the $\mathrm{CBC}$ examination were within normal limit except for the high erythrocyte sedimentation rate (ESR). The ESR was $49 \mathrm{~mm} / \mathrm{hr}$ that was increased by 20 points compared to the normal limit. Oral hygiene instruction was given and follows up observation for the lesion was scheduled in two weeks time.

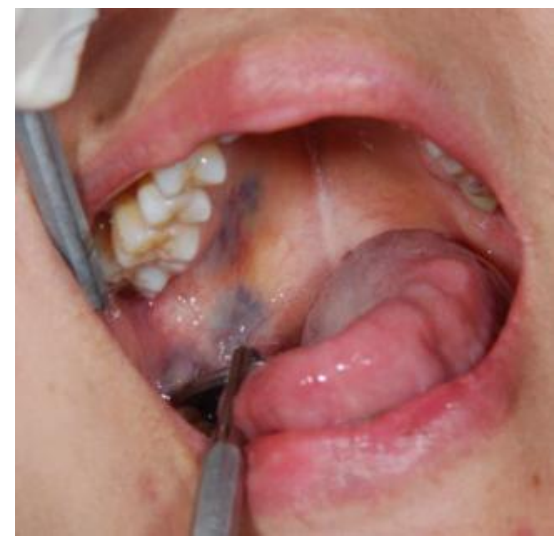

Figure 1. Clinical features of focal melanoticlesions: irregular flat dark blue macula in the hard and soft palate, and purplish blue nodules retromolar pad area of 48 tooth

The maxillary occlusal radiograph showed no pathological signs/signs of bone destruction. Radiographic interpretations were 36 filling extend to pulp chamber and 46 post endodontic treatments, appear pathological chronic periapical with separation of the lamina durra. No evidence of bone destruction/pathological hallmark of the hard tissues of the maxilla and mandible (Figure 2). The OPG showed that the teeth shape of the maxilla and mandible were within the normal range, 36 dental filling reaches the pulp chamber; there was a pathological chronic periapical radiolucent. The 46 post-treatment endodontic appeared a pathological chronic periapical that is periapical-periodontal junction widening with the separation of lamina durra. The alveolar bone height of the maxilla and the mandibular were normal. Anatomical structures and surrounding appeared in the normal range (Figure 3).

On the follow-up visit, the bluish macular lesions were located on the border of the posterior hard palate to the soft palate showed increased color intensity when compared to the previous appearance. The lesions looked increased in the vascularity and dimension (Figure 4). The size of the lesions was increased by $2 \mathrm{~mm}$. The lesion was not tender on palpation. An incisional biopsy and histopathological assessment of the lesion were mandatory for making a definitive diagnosis.

The patient decided to use the National Health Security System to cover the treatment related to the lesion since the concerns of high cost of therapy. According to the system, the patient needed to get a referral letter from the Primary Health Facility to the General Hospital which would do the biopsy and histopathology. The dentist of the Primary Health Facility needs to make the suspected diagnosis and refer it to the National General Hospital. 

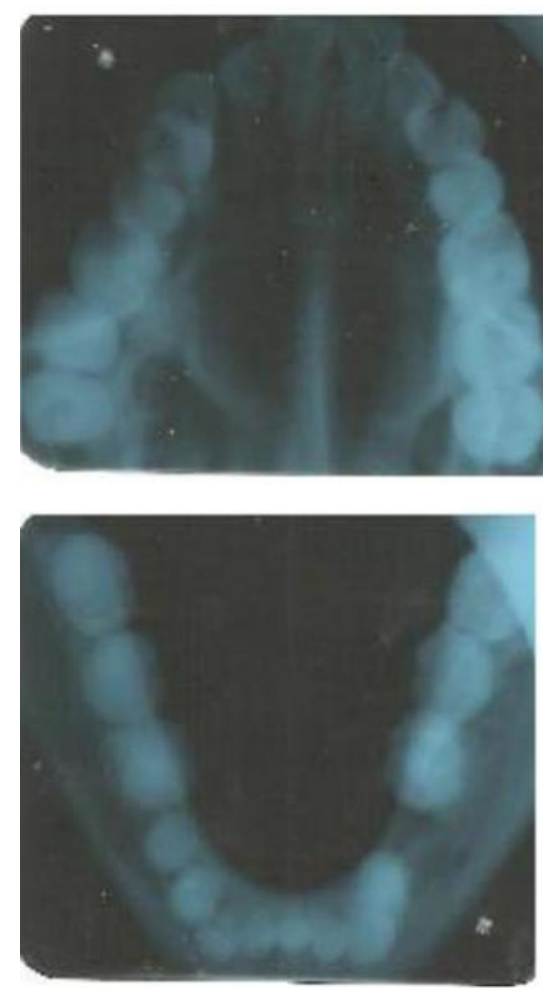

Figure 2. Occlusal radiographs showed nopathological signs of bone destruction

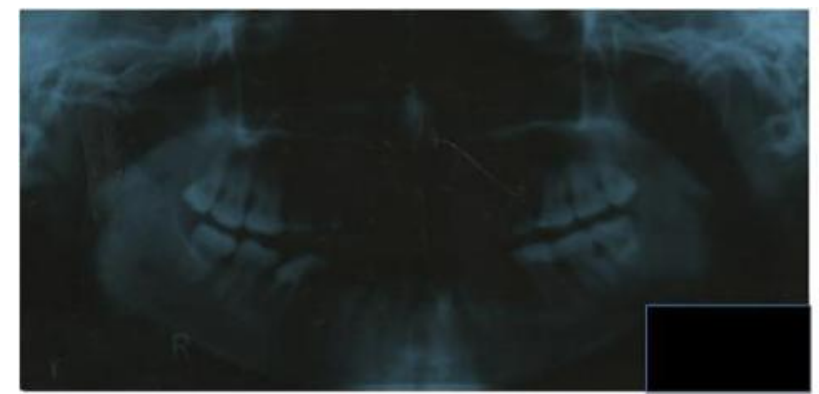

Figure 3. The panoramic radiograph showed thenormal condition of the maxilla and mandibula bone, bone destruction or hallmark of pathological condition was not found

Until this case report was written, we had not received the results of her biopsy yet. In fact, she did not get a referral letter from the dentist at the Primary Health Facility for further treatment of her lesion. The dentist stated that there was no indication for biopsy to the lesion on her palatal mucosa. The lesion was not something to worry about since it was asymptomatic in nature. The patient decided for not having any treatment for the lesion.

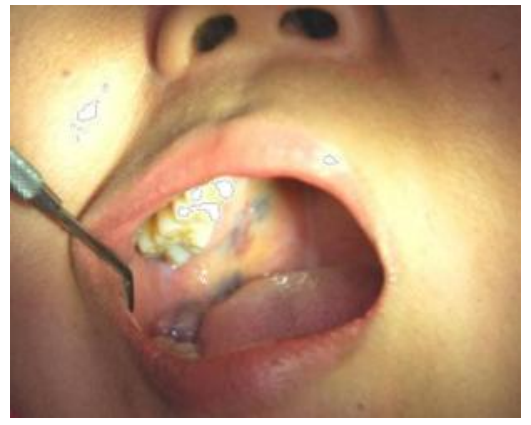

Figure 4. Clinical feature of the lesions in the following month. The dark blue macula in the hard and soft palate, irregular, flat, with purplish blue nodules retromolar pad area of $\mathbf{4 8}$ teeth were still existed. The color intensity and the size of the lesions were markedly increased

\section{Discussion}

The clinical diagnosis of pigmented lesions is usually made by size, shape, color and the presented clinical information. However, a definitive diagnosis of pigmented lesion in the oral mucosa could be challenging, since the definitive clinical criteria of each lesion have not been available. Therefore, in some cases, the histopathology and sometimes immunohistochemistry examination may be required. ${ }^{3,5}$

In order to help clinicians to do a diagnostic pathway when finding pigmented lesions in the oral cavity, a flowchart for diagnosis and management of oral pigmented lesion had been proposed (Figure 5). ${ }^{2}$ When ones have classified the lesion to be melanocytic-related, clinical suspicion of malignancy in terms of ABCD checklist (asymmetry, border irregularities, color variegation and diameter $>6 \mathrm{~mm}$ ), recent history, age and the involved subsites should be assessed. ${ }^{2}$ The diagnosis of lesions with "no or low suspicion of malignancy" such as oral manifestation of systemic disease, physiologic pigmentation, smoker's melanosis, may be made based on clinical information alone or may need histopathological assessment. ${ }^{2,3,5}$

Eral, the pigmented lesions in the oral cavity are benign in nature, which required no treatment unless discomfort is present. However, special concerns have to be put on OMM. The OMM is an aggressive melanocytesoriginated tumor, which accounted for less than $1 \%$ of all malignancy in the oral cavity. ${ }^{7}$ It is a rare tumor, and the definite etiology and pathogenesis are unknown. Some reports have shown that the clinical differentiation between OMM and melanotic macules, oral melanotic nevus or amalgam tattoos could be challenging. It was reported that OMM could suddenly arise from clinically healthy mucosa or in approximately around $30 \%$ of the OMM is initially presented as asymptomatic oral pigmentation for months or years before they were emerged as mass lesions and becoming symptomatic. ${ }^{6-9}$ The palatal and maxillary gingival mucosa are the most common sites of OMM. Therefore, the presence of pigmented lesions in this area should increase suspicion to OMM. ${ }^{10-13}$ 


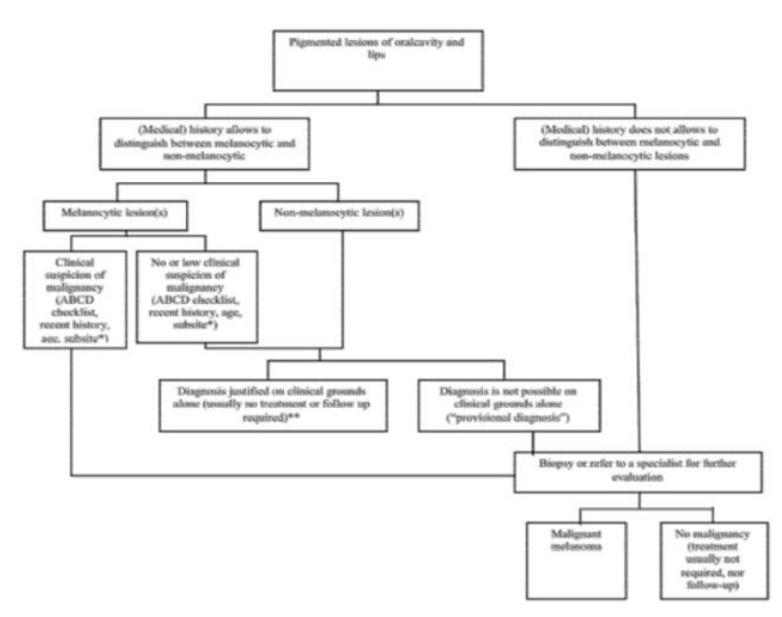

Figure 5. A flow-chart for diagnosis and management of oral pigmented lesion. (* Palate and gingiva are considered subsite at risk for OMM development; ** In case of multiple or diffuse pigmentation(s) further examination for underlying diseases or syndromes (e.g. Addison's, PeutzJeghers syndrome, etc.) may be considered). ${ }^{2}$

The patient in this case report was referred to our clinic by her family dentist (private practitioner) who found an asymptomatic pigmented lesion on the palatal mucosa. After careful history taking, $\mathrm{CBC}$ and radiograph examination was performed. The ABCD checklist was also done. ${ }^{14} \mathrm{We}$ found asymmetry, border irregularities, color variegation (blue, purple, red) and diameter $>6 \mathrm{~mm}$ in the lesion. The lesion had been asymptomatic and slow growing for ten years, and they were located on the palatal and gingival mucosa where the suspicion to OMM should be increased. Furthermore, the history of the underlying disease or syndrome could not be found. Based on the clinical assessment of the lesion, the provisional diagnosis of OMM was made. An incisional biopsy for histopathological examination is mandatory to make a definitive diagnosis. ${ }^{2}$ However, there were obstacles to complete full diagnosis pathway of this pigmented lesion in the patient. The system of patient referral for treatment in the National Hospital using National Health Security System complicated the process. The patient needed to get a reference letter for biopsy from a general dental practitioner from a Primary Health Facility to the National General Hospital. However, the asymptomatic nature of the lesion making the dentist in the facility was unaware of the importance of biopsy for making the diagnosis. The situation had made the patient lost interest to have the lesion being examined. Until this case report was written, the patient had not decided to undergo biopsy. A followup visit was then encouraged to further observe the lesion since there was a report of delayed diagnosis of OMM which previously was thought to be a racial pigmentation. ${ }^{9}$ The diagnosis of racial pigmentation on the previous report was only based on the clinical aspect of the lesion. Based on the experience from this case, the dentist's awareness regarding the possible finding of potentially malignant pigmented lesion with asymptomatic nature still needs to be increased.

Many reports have described vast variation in the clinical presentations of OMM, from uniformly brown or black to only black, brown, gray, purple, reddish or even without pigmentation. Furthermore, its appearance could be complicated by the presence ulceration and bleeding. ${ }^{2,9,12}$ The examination on the lesion's ABCD checklist (asymmetry, border irregularities, color variegation and diameter $>6 \mathrm{~mm}$ ) as for cutaneous melanoma could also be used for the clinical diagnosis of OMM. ${ }^{14}$ The growth characteristics of OMM could be initiated by long period of radial growth phase followed by vertical growth phase or in other types aggressive vertical growth phase could start from the beginning of the disease. ${ }^{3,15}$

Histopathologically, OMM may show a degree of pleomorphism with a wide variation of shapes and patterns which may be similar to other types of tumors such as lymphomas, poorly differentiated carcinomas, sarcomas, neuroendocrine carcinomas and germ-cell tumors. ${ }^{9,12}$ The complexity of the disease is added by the fact that some OMM may be nonpigmented, making the clinical diagnosis becoming more difficult. In such cases, the immunohistochemistry analysis using S-100, HMB45, Melan-A, and vimentin are needed to diagnose the disease accurately. ${ }^{12}$

The treatment for OMM involves radical surgical excision to include all satellites lesion should be done to gain tumor-free margin. The surgery must have a wide margin and bone resection. ${ }^{11}$ It is sometimes difficult to gain the tumor-free margin condition because of involvement of vital anatomical structures. ${ }^{3}$ The OMM is not radiation and chemotherapy sensitive.

Therefore, those treatments are ineffective. ${ }^{3}$ The occurrence of distant metastases of OMM is found in the lungs, brain, liver or bones. These complex nature of the disease makes the OMM has poor prognosis, which accounts for only $15 \%$ overall 5 -year survival rate. ${ }^{12}$

\section{Conclusions}

The diagnosis of pigmented lesion in the oral cavity could be quite challenging. Sometimes the clinical aspects of the pigmented lesions may be sufficient to conclude a diagnosis. However, in some cases differentiation between some low suspicion pigmented lesion, and OMM could not be made clinically, making biopsy for histopathology with or without immunohistochemistry are needed. The dentist should be aware of the presence of the pigmented lesions in the patients' oral mucosa and carefully undergo a diagnostic pathway to come to the definitive diagnosis.

\section{References}

1. Kauzman A, Pavone M, Blanas N, Bradley G. Pigmented lesions of the oral cavity: review, differential diagnosis, and case presentation. J Can Dent Assoc. 2004;70:682-3.

2. Meleti M, Vescovi P, Mooi WJ, van der Waal I. Pigmented lesions of the oral mucosa and perioral tissues: a flow-chart for the diagnosis and some recommendations for the management. Oral Surg Oral Med Oral Pathol Oral Radiol Endod. 2008;105(5):606-16.

3. Gondak RO, da Silva-Jorge R, Jorge J, Lopes MA, Vargas PA. Oral pigmented lesions: Clinicopathologic 
features and review of the literature. Med Oral Patol Oral Cir Bucal. 2012;17(6):e919-24.

4. Mergoni G, Ergun S, Vescovi P, Mete Ö, Tanyeri H, Meleti M. Oral postinflammatory pigmentation: an analysis of 7 cases. Med Oral Patol Oral Cir Bucal. 2011;16(1):e11-4.

5. Reddy BV, Sridhar GR, Anuradha $\mathrm{CH}$, Chandrasekhar P, Lingamaneni KP. Malignant melanoma of the mandibular gingiva: a rare occurrence. Indian $J$ Dent Res. 2010;21(2):302-5.

6. Rapini RP, Golitz LE, Greer Jr RO, Krekorian EA, Poulson T. Primary malignant melanoma of the oral cavity. A review of 177 cases. Cancer 1985;55:1543-51.

7. Meleti M, Leemans CR, Mooi WJ, Vescovi P, van der Waal I. Oral malignant melanoma: a review of the literature. Oral Oncol 2007;43: 116-121.

8. Gondivkar SM, Indurkar A, Degwekar S, Bhowate R. Primary oral malignant melanoma--a case report and review of the literature. Quintessence Int. 2009;40(1):41-6.

9. Martinelli-Kläy CP, Laporte ML, Martinelli CR, Martinelli C, Lombardi Oral Malignant Melanoma Initially Misdiagnosed as a Racial Pigmentation: A Case Report. Dermatopathology (Basel). 2016;3(1):1-7.
10. Meleti M, Leemans CR, Mooi WJ, van der Waal I. Oral malignant melanoma: the amsterdam experience. Oral Maxillofac Surg. 2007;65(11):2181-6.

11. Aguas SC, Quarracino MC, Lence AN, Lanfranchi-Tizeira HE. Primary melanoma of the oral cavity: ten cases and review of 177 cases from literature. Med Oral Patol Oral Cir Bucal. 2009 ;14(6):E265-71.

12. de-Andrade BA, Toral-Rizo VH, León JE, Contreras E, Carlos R, Delgado-Azañero W, Mosqueda-Taylor A, deAlmeida OP. Primary oral melanoma: a histopathological and immunohistochemical study of 22 cases of Latin America. Med Oral Patol Oral Cir Bucal. 2012;17:383-8.

13. Kumar V, Vishnoi JR, Kori CG, Gupta S, Misra S, Akhtar N. Primary malignant melanoma of oral cavity: A tertiary care center experience. Natl $J$ Maxillofac Surg. 2015;6(2):167-71.

14. Younes MN, Myers JN. Melanoma of the head and neck: current concepts in staging, diagnosis, and management. Surg Oncol Clin North Am. 2004;13:201-29.

15. Sharma N. Primary oral malignant melanoma: two case reports and review of literature. Case Rep Dent. 2012;2012:975358. 